\title{
Descripción de un caso clínico de Leishmaniasis canina
}

\author{
Cabrera, A.M.; Betancourt, D.A.; Carrillo, N.G. \\ Facultad de Medicina Veterinaria y Zootecnia, Universidad Central del Ecuador \\ E-mail: dabetancourt@uce.edu.ec
}

\begin{abstract}
Resumen
Cabrera, A.M.; Betancourt, D.A.; Carrillo, N.G.: Descripción de un caso clínico de Leishmaniasis canina. Rev. Vet. 32: 2, 242-245, 2021. La leishmaniasis es una enfermedad parasítica ocasionada por un protozoario del género Leishmania, propagada a través de la picadura de insectos contagiados de la familia Phlebotomidae. Conforme menciona la Organización Mundial de la Salud, se cree que su incidencia aumenta conforme pasan los años. La afección es de tipo zoonótica. La patología y epidemiología de leishmaniasis puede mostrarse dependiendo de la especie del parásito, de las tipologías ecológicas de las zonas en donde se transmite y de la exposición anterior y presente ante el parásito. Por ello se presentará una forma visceral conocida como kala azar teniendo una alta mortalidad al no tratarla, ya que posee la capacidad de provocar brotes que conlleven a la muerte, caracterizada por la presencia de sucesos anormales de pirexia, disminución de peso, anemia y hepato-esplenomegalia. Una de las formas más frecuentes es la leishmaniasis cutánea, que provoca úlceras a nivel de la piel, las que son causantes de incapacidad grave; la leishmaniasis mucocutánea induce la pérdida parcial o total de las mucosas del área de la nariz, boca y garganta. Se han hallado casos de leishmaniasis en la mayoría de los países, tanto en regiones subtropicales como tropicales o de climas mediterráneos, ya que la presencia de la familia Phlebotomidae es alta. Con relación a los diagnósticos empleados para la identificación de leishmaniasis, se aplican técnicas morfológicas o técnicas de detección antigénica.
\end{abstract}

Palabras clave: leishmaniasis, protozoario, phlebotomidae, zoonosis.

\begin{abstract}
Cabrera, A.M.; Betancourt, D.A.; Carrillo, N.G.: Description of a clinical case of canine leishmaniasis. Rev. Vet. 32: 2, 242-245, 2021. Leishmanias is a disease considered parasitic caused by a protozoan of the genus Leishmania, spread through the bite of infected insects of the Phlebotomidae family. As mentioned by the World Health Organization, it is believed that its incidence is high as the years go by zoonotic-ty pe disease. The pathology and epidemiology of leishmaniasis can be shown depending on the sp of the parasite, of the ecological typologies of the areas where it is transmitted, and of the previous and present exposure to the parasite. Therefore, it occurs in a visceral form known as kalaazar, having a high mortality rate when not treated, since it can cause outbreaks that lead to death, characterized by the presence of abnormal events of pyrexia, weight loss, anemia, and hepatosplenomegaly. One of the most frequent forms is leishmaniasis is the cutaneous one which causes ulcers at the cutaneous level, which are the cause of serious disability; mucocutaneous leishmaniasis which induces the partial or total loss of the mucous membranes of the nose, mouth, and throat area. Cases of leishmaniasis have been found in most countries, with the following factors such as subtropical and tropical regions or Mediterranean climates, since the presence of the Phlebotomidae family is high, migratory populations due to the incidence that it entails. In relation to the diagnostic techniques used for the identification of leishmaniasis, morphological techniques or antigenic detection techniques are applied. Leishmanias is a disease considered parasitic caused by a protozoan of the genus Leishmania, spread through the bite of infected insects of the Phlebotomidae family. As mentioned by the World Health Organization, it is believed that its incidence is high as the years go by. zoonotic-type disease. The pathology and epidemiology of leishmaniasis can be shown depending on the sp of the parasite, of the ecological typologies of the areas where it is transmitted and of the previous and present exposure to the parasite. Therefore, it occurs in a visceral form known as kalaazar, having a high mortality rate when not treated, since it can cause outbreaks that lead to death, characterized by the presence of abnormal events of py rexia, weight loss, anemia, and hepato-splenomegaly. One of the most frequent forms is Leishmaniasis is the cutaneous one which causes ulcers at the cutaneous level, which are the cause of serious disability; Muco-cutaneous leishmaniasis which induces the partial or total loss of the mucous membranes of the nose, mouth, and throat area. Cases of
\end{abstract}


leishmaniasis have been found in most countries, with the following factors such as subtropical and tropical regions or Mediterranean climates, since the presence of the Phlebotomidae family is high, migratory populations due to the incidence that it entails. In relation to the diagnostic techniques used for the identification of leishmaniasis, morphological techniques or antigenic detection techniques are applied.

Key words: leishmaniasis, protozoan, phlebotomidae, zoonotic.

\section{INTRODUCCIÓN}

La leishmaniosis es una enfermedad transmitida por vectores a los seres humanos y animales. Es causada por parásitos protozoarios del género Leishmania. Los caninos son susceptibles de adquirir las formas visceral y cutánea y son los principales reservorios de Leishmania infantum, el agente causal de la forma visceral en seres humanos ${ }^{1}$

Las manifestaciones consisten en síndromes cutáneos, mucosos y viscerales. La leishmaniasis de piel causa lesiones cutáneas (LC) crónicas indoloras, que van desde nódulos hasta grandes úlceras capaces de persistir durante meses o años, aunque por último cicatrizan ${ }^{4}$.

La enfermedad produce diversas alteraciones inmunológicas en el perro, como la supresión de la inmunidad celular. Ella genera la multiplicación incontrolada del parásito en el citoplasma del macrófago y la activación de la inmunidad humoral, que origina una producción elevada de inmunoglobulinas, así como la formación de inmunocomplejos circulantes, cuyo depósito puede conducir a vasculitis y glomerulonefritis ${ }^{2}$.

En la patogenia del perro y del ser humano existe un mecanismo inmunopatogénico muy importante en el avance de la enfermedad. Debido al desarrollo de inmunocomplejos, éstos se depositan en distintos órganos, como bazo, hígado, riñón y -de manera sistémicaen los vasos sanguíneos, propiciando distintos cuadros clínicos característicos de la enfermedad.

En los perros la forma visceral induce un cuadro clínico variable con manifestaciones tanto cutáneas como viscerales, sin embargo, del 35 al 59\% de los animales infectados son asintomáticos, lo cual plantea un importante problema de salud pública ${ }^{9}$. Una de las pocas diferencias es la presencia de lesiones cutáneas superficiales en perros, las cuales resultan raramente advertidas en seres humanos. El periodo de incubación de la LC es variable. Se han notificado intervalos entre 2 y 25 meses en formas subclínicas de LC experimental ${ }^{3}$.

Los animales infectados de forma natural presentan periodos asintomáticos prolongados. En Europa la LC representa un serio problema y se calcula que alrededor de siete millones de perros están en riesgo de padecer la enfermedad ${ }^{8}$.

En esta afección son muchas las alternativas diagnósticas, pero su sensibilidad y especificidad, así como su relación costo/beneficio, resultan variables. Sin duda, la forma más contundente de diagnosticar leishmaniasis es demostrar la presencia del parásito; sin embargo, los procedimientos poseen amplios márgenes de sensibilidad y especificidad. Por ejemplo, el diagnóstico parasitológico es específico $(100 \%)$, pero su sensibilidad varía de 50 a $60 \%$ en cultivos de aspirados de ganglios linfáticos y de 60 a $80 \%$ en aspirados de médula ósea ${ }^{3}$.

El objetivo del presente trabajo fue reportar los resultados de los exámenes realizados para el diagnóstico del paciente, además de los hallazgos parasitológicos e histopatológicos realizados en la necropsia.

\section{MATERIAL Y MÉTODOS}

Se presentó a consulta en la ciudad de Quito (Ecuador), un perro macho de cinco años, raza Pitbull. El animal exhibió un cuadro grave de depresión, hiporexia, anorexia, pérdida de peso, problemas cutáneos y dificultades visuales.

Las constantes fisiológicas del animal estaban dentro de los rangos normales, a excepción de una hipertermia $\left(39,3^{\circ} \mathrm{C}\right)$, mientras que el examen físico presentaba adenopatía generalizada y trastornos dérmicos como: eritema, alopecias, costras y escoriaciones en la punta de las orejas, además una postura acompañada de cojera.

Se establecieron los siguientes diagnósticos diferenciales: leishmaniosis, demodicosis, dermatofitosis, pénfigo foliáceo y ehrlichiosis. Para establecer el diagnóstico verdadero se realizaron los siguientes exámenes: biometría hemática, química sanguínea y serología contra Ehrlichia canis. También se empleó un paquete diagnóstico para detectar anticuerpos anti-E.canis y se realizaron raspados cutáneos y citología de ganglios linfáticos.

Los dueños del animal decidieron que se le practicara eutanasia, la cual se realizó por sobredosis de barbitúricos, tras lo cual se practicó la necropsia. Durante esta última se registraron las alteraciones más importantes, tomando muestras de los órganos para realizar estudios histopatológicos y parasitológicos.

Para detectar posibles parásitos, se realizaron frotis de bazo, hígado y ganglio linfático poplíteo. Una gota de la muestra tomada de cada órgano fue depositada por separado sobre un portaobjeto. Posteriormente, con la ayuda de otro portaobjetos limpio, se extendió la muestra y se dejó secar al aire, para luego fijarla con metanol al 95\%.

Estas muestras se tiñeron con Diff Quick ${ }^{\circledR}$. Además, se tomaron muestras de bazo, hígado, ganglio linfático poplíteo y médula ósea, que fueron vertidas en el interior de tubos con medio Novy-MacNeal-Nicolle 
$(\mathrm{NNN})$, colocándose en incubación a $27^{\circ} \mathrm{C}$ y examinándose una vez por semana.

Con la finalidad de realizar los estudios histopatológicos, luego de su sacrificio se tomaron muestras del animal de hígado, bazo, ganglio linfático poplíteo y piel, todos los órganos fueron pasados a solución de formaldehído al $10 \%$ en PBS, realizándose cortes en dirección al hilio de cada órgano para que la solución conservadora penetrara en los tejidos.

Las muestras de cada órgano se incluyeron en parafina mediante procesamiento automático. Luego se realizaron cortes de $5 \mu \mathrm{m}$ para obtener laminillas que se tiñeron mediante la técnica de hematoxilina-eosina para su ulterior análisis histopatológico.

\section{RESULTADOS}

Análisis diagnósticos. Los resultados fueron: leucocitosis por neutrofilia con desviación a la izquierda, anemia, azotemia e hiperproteinemia; el resultado de la serología contra $E$. canis resultó negativo; el raspado cutáneo fue negativo a ácaros cutáneos, finalmente, la citología -tanto de ganglios linfáticos poplíteos como de médula ósea- reveló presencia de amastigotes de Leishmania sp, de dos por cuatro puntos cinco micras.

Resultados parasitológicos. En todos los frotis realizados a partir de bazo, hígado, ganglio linfático poplíteo y médula ósea se apreciaron amastigotes de Leishmania $s p$, mientras que la mayoría de los tubos de NNN inoculados se contaminaron con bacterias, pero en un tubo que contenía muestras de médula ósea se logró apreciar el desarrollo de promastigotes de Leishmania $s p$ con moderada motilidad.

Resultados de la necropsia del perro. Piel: lesiones cutáneas generalizadas caracterizadas por dermatitis exfoliativa y escoriaciones en piel, además se observaron zonas de alopecia en el tabique nasal y periferia de los ojos. Mucosas: palidez de mucosas. Ojos: se detectó conjuntivitis, uveítis y blefaritis. Músculos: palidez en masas musculares, así como atrofia de los músculos masticatorios y de la cabeza. Ganglios linfáticos: se observó inflamación generalizada de todos los nódulos linfáticos, en particular los poplíteos, inguinales y submaxilares. Articulaciones: inflamación de las articulaciones del codo, coxofemoral y femurorotuliana. Hígado: hepatomegalia, congestionado y con bordes redondeados. Riñones: ligeramente fibrosos al corte. Bazo: esplenomegalia, congestionado y con bordes redondeados.

Resultados del análisis histopatológico. En piel se observó un infiltrado mixto formado de macrófagos, linfocitos, células plasmáticas y presencia de parásitos. A nivel de encéfalo se observaron áreas hemorrágicas en sustancia blanca. En corazón hubo degeneración hialina y necrosis de fibras cardiacas. En bazo se observó microscópicamente que la pulpa blanca se pre- sentó atrofiada. En el hígado se observó hipertrofia e hiperplasia de las células de Kupffer, con presencia de parásitos en dichas células, así como de granulomas, también se observó necrosis individual de hepatocitos, además de retención de bilis. En ganglios linfáticos se apreció que los centros germinales estaban destruidos y los folículos no se formaron correctamente. Los macrófagos estaban parasitados. En la médula se observó un fuerte infiltrado de células plasmáticas, abundante hemosiderina contenida en macrófagos, y también se presentó hiperplasia de sus elementos. En riñón hubo distensión y congestión glomerular con depósitos de material proteínico en el espacio de Bowman; además se observó degeneración y necrosis de células del epitelio de túbulos. En pulmón se presentó neumonía intersticial con infiltrado mononuclear en las paredes alveolares y hemorragias pulmonares; también se observaron áreas moderadas de atelectasia y enfisema.

\section{DISCUSIÓN}

De acuerdo con los hallazgos obtenidos en los estudios parasitológicos e histopatológicos, las lesiones corresponden a una afectación severa de la enfermedad en el paciente, es decir, hubo degeneración y no se pudo instaurar tratamiento. Algunas de las lesiones eran comunes y otras se presentaron en raras ocasiones.

Las encontradas en piel y ojos eran comunes en caninos y se presentaron con engrosamiento cutáneo en forma de placas y pápulas ${ }^{1}$. En las articulaciones puede provocar lesiones, que aparecen como manifestación de una reacción de hipersensibilidad tipo III, en la cual las enzimas liberadas por las células inflamatorias, sinoviocitos y condrocitos, dañan al cartílago articular fomentando la aparición de anormalidades erosivas ${ }^{5}$.

Los animales con una adecuada respuesta inmunitaria celular van a poder eliminar el parásito de leishmania ${ }^{6}$. Si esta respuesta inmune no es adecuada, el parásito invadirá células como los macrófagos y otras del sistema inmunitario donde se multiplicará para después invadir la médula ósea, ganglios linfáticos, bazo e hígado ${ }^{7}$. Entonces se desarrollará una respuesta humoral en la que se fabricará gran cantidad de anticuerpos.

Las lesiones presentadas en la musculatura, son producidas por los amastigotes encontrados dentro del tejido; esta afectación puede producir una alteración en la alimentación y por ende ocurrirá el adelgazamiento y la atrofia muscular ${ }^{10}$.

Actualmente no existe ninguna vacuna efectiva frente a la leishmaniosis, por lo cual la solución para evitar que un perro se contagie, pasa por evitar o disminuir el contacto con el vector, ya que no hay un método realmente efectivo que asegure una protección completa frente a Leishmania sp.

Agradecimientos. Al Docente F.A.Pazmiño (Universidad Central del Ecuador) porque su enseñanza y orientación permitieron diseñar las bases primordiales del área estudiada. 


\section{REFERENCIAS}

1. Acero VP et al. 2015. Canine leishmaniosis: tools for diagnosis in veterinary practice in Colombia. Rev Med Vet Zoot Córdoba 20: 3, 4822-4842.

2. Aragón JE, Gómez FJ. 2010. Leishmaniosis canina y humana. Rev Epidemiol 1: 1, 1-6.

3. Facultad de Veterinaria, Universidad Complutense de Madrid. 2018. Leishvet: Leishmaniosis canina y felina, 18: 14.

4. Filho D, Vedobello FA, Jorge MV, Lonardoni UT. 2010 Leishmaniasis cutánea y visceral. Man diagnostic tests vaccines Terr Anim 2: 10, 1-13.

5. Lockett MB, Llano EG, Maidana HR, Báez AD, Cabrera WR. 2019. Poliartritis asociada a leishmaniasis en un canino del nordeste argentino. Rev Vet 30: 1, 28-31.

6. Portus M. 1990. Leishmaniosis canina. Enferm Infecc Microbiol Clin 8: 4, 251.
7. Rivero ME, Rodríguez JL, Pérez A, Bejarano EE. 2021 Isolation of Leishmania infantum from canis familiaris in an urban area of the colombian caribbean. Rev Investig Vet Peru 29: 3, 923-930.

8. Rodríguez BS et al. 2012. Situación epidemiológica y de los factores de riesgo de transmisión de Leishmania infantum en España. Rev Esp Salud Publ 86: 6, 555-564.

9. Vargas JJ, López MC, Escovar JE, Fernández MJ. 2009. Evaluación por Western Blot, inmunofluorescencia indirecta y ELISA de perros infectados con Leishmania infantum. Rev Salud Publ 11: 4, 641-652.

10. Villamizar R, Irlene E. 2009. Respuesta inmune a la infección por Leishmania infantum en caninos (Immune response to infection by Leishmania infantum in dogs). Redvet X: 1-8. 